\title{
Radiogenomic analysis of vascular endothelial growth factor in patients with diffuse gliomas
}

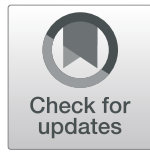

\author{
Zhiyan Sun ${ }^{1 \dagger}$, Yiming $\mathrm{Li}^{1+}$, Yinyan Wang ${ }^{2}$, Xing Fan ${ }^{1}$, Kaibin Xu ${ }^{3}$, Kai Wang ${ }^{4}$, Shaowu $\mathrm{Li}^{1}$, Zhong Zhang ${ }^{2}$, \\ Tao Jiang ${ }^{1,2,5,6,7}$ and Xing Liu ${ }^{1^{*}}$ (D)
}

\begin{abstract}
Objective: To predict vascular endothelial growth factor (VEGF) expression in patients with diffuse gliomas using radiomic analysis.

Materials and methods: Preoperative magnetic resonance images were retrospectively obtained from 239 patients with diffuse gliomas (World Health Organization grades II-IV). The patients were randomly assigned to a training group $(n=160)$ or a validation group $(n=79)$ at a 2:1 ratio. For each patient, a total of 431 radiomic features were extracted. The minimum redundancy maximum relevance (mRMR) algorithm was used for feature selection. A machine-learning model for predicting VEGF status was then developed using the selected features and a support vector machine classifier. The predictive performance of the model was evaluated in both groups using receiver operating characteristic curve analysis, and correlations between selected features were assessed.

Results: Nine radiomic features were selected to generate a VEGF-associated radiomic signature of diffuse gliomas based on the mRMR algorithm. This radiomic signature consisted of two first-order statistics or related wavelet features (Entropy and Minimum) and seven textural features or related wavelet features (including Cluster Tendency and Long Run Low Gray Level Emphasis). The predictive efficiencies measured by the area under the curve were $74.1 \%$ in the training group and $70.2 \%$ in the validation group. The overall correlations between the 9 radiomic features were low in both groups.
\end{abstract}

Conclusions: Radiomic analysis facilitated efficient prediction of VEGF status in diffuse gliomas, suggesting that using tumor-derived radiomic features for predicting genomic information is feasible.

Keywords: Vascular endothelial growth factor, Diffuse gliomas, Radiomic analysis, Machine learning

\section{Introduction}

Diffuse gliomas graded from II to IV according to the World Health Organization (WHO) criteria are the most common primary malignant tumors of the brain [1]. Surgical resection combined with radiotherapy and chemotherapy are the main treatments. With the development of precision medicine, the molecular classification of gliomas has become increasingly important with regard to the treatment and prognosis of patients. In 2016, the WHO

\footnotetext{
* Correspondence: 15846591696@126.com

†Zhiyan Sun and Yiming Li contributed equally to this work.

${ }^{1}$ Beijing Neurosurgical Institute, Capital Medical University, 6 Tiantanxili, Beijing 100050, China

Full list of author information is available at the end of the article
}

introduced molecular phenotyping into the classification criteria of tumors of the central nervous system. Accordingly, it is now important to determine the molecular subtype of gliomas prior to treatment $[2,3]$.

Angiogenesis, which is regulated by vascular endothelial growth factor (VEGF), is a marker of the malignancy of tumor cells. Tumor cells with high expression of VEGF often result in poor prognosis and short survival [4]. In addition, VEGF is a well-known biomarker that is of great significance in the development of tumors, and it is also a promising target in the treatment of gliomas, especially recurrent glioblastomas (GBM) [4-6]. Although antiangiogenic therapies, such as bevacizumab, have been proved to increase progression-free survival in patients with

(C) The Author(s). 2019 Open Access This article is distributed under the terms of the Creative Commons Attribution 4.0 International License (http://creativecommons.org/licenses/by/4.0/), which permits unrestricted use, distribution, and 
recurrent GBM, it may not be beneficial for unselected patients [7]. Therefore, the evaluation of VEGF expression held great promising in GBM management.

Magnetic resonance imaging (MRI) is widely used clinically, particularly for the non-invasive imaging of tumors, and it has become one of the most commonly utilized methods for the diagnosis of central nervous system tumors [3, 8]. Notably however, current MRI applications can only analyze the physicochemical characteristics of tumors qualitatively $[9,10]$. This ignores a large amount of the digital information in the image. Radiomics is a noninvasive method for extracting textural information from radiological images for analysis and calculation [10-13]. Some studies have applied radiomics technology to tumor analysis and have shown that the approach is feasible [1416]. Some researchers made a convolutional neural network to determine the Isocitrate dehydrogenase (IDH) mutation status [17], 1p/19q codeletion, and O6methylguanine-DNA methyltransferase (MGMT) promotor methylation status [18]. Some researchers found the relationship between epidermal growth factor receptor (EGFR) extracellular domain missense mutations and clinical imaging and therapeutic response [19], and established an imaging signature of EGFRvIII [20]. These radiomics approaches have been successfully utilized to predict the genotype of IDH mutation, the expression of EGFR and Ki67 in gliomas [21-23], laying the foundation for detecting VEGF expression status non-invasively.

In the current study, we extracted a large number of radiomics features from preoperative MRI scans of glioma patients with known VEGF expression levels. We hypothesized that a radiomic signature could predict the level of VEGF expression in gliomas via a machine-learning algorithm.

\section{Materials and methods Patients}

A total of 239 patients with gliomas were included in this study. All the patients received treatment at Beijing Tiantan Hospital glioma therapy center between June 2010 and September 2012, and met the following criteria: (a) histopathologically confirmed primary glioma, WHO grade II-IV; (b) preoperative T2-weighted magnetic resonance images available; (c) VEGF expression status known; and (d) specific clinical characteristics known (see Additional file 1: Figure S1). A flowchart depicting the exclusion and inclusion of patients is shown in Additional file 1: Figure S1.

The patients were randomly assigned to a training group $(n=160)$ or a validation group $(n=79)$, regardless of VEGF expression level. The random process was performed using the random number generated from the $\mathrm{R}$ software. The training group was used to establish a machine-learning model to predict the level of VEGF expression via radiomics features, while the validation group was used to assess the prediction accuracy of the model. Ethics approval for this retrospective study was obtained from the institutional review board of Beijing Tiantan Hospital.

\section{Clinical characteristics}

The median ages of the patients were 43.0 years in the training group and 42.5 years in the validation group. There were 157 males and 82 females in total, and via random assignment 104 men and 56 women were allocated to the training group and 53 men and 26 women were allocated to the validation group. The proportions of patients with low and high VEGF expression were 63/ 97 in the training group and 27/52 in the validation set. The distributions of characteristics in the two groups were compared using Student's $t$-test and the Chi-square test, and there were no significant differences in age $(p=0.736)$, sex $(p=0.749)$, tumor grade $(p=0.725)$, VEGF expression level $(p=0.435)$, or tumor location $(p=0.860)$. Detailed information pertaining to the clinical characteristics of the patients is shown in Table 1.

\section{Data acquisition and region of interest segmentation}

MRI scans were performed using 3.0-T scanners (179 patients: Trio, 3.0-T, Siemens; 60 patients: sigma, 3.0-T, GE). Tumor regions of interest (ROIs) were only segmented on T2-weighted (T2) images because II-IV grades of gliomas were included in the current study (identifying tumor borders of low-grade gliomas is hard on T1-weighted and contrast-enhanced sequences). The parameters used to acquire T2 images were repetition time $4500 \sim 6000 \mathrm{~ms}$, echo time $84 \sim 122.5 \mathrm{~ms}$, section thickness $3 \sim 5 \mathrm{~mm}$, field of view of $(180 \sim 240) \mathrm{mm} \times$ $(219 \sim 256) \mathrm{mm}$, and matrix size $(160 \sim 512) \times(208 \sim 512)$ pixels. ROIs were manually delineated by two neuroradiologists (with eight- and 10-year work experience in the field of neuroradiology, respectively) on T2 images using the MRIcron software (http://www.mccauslandcenter.sc. edu/mricro). ROIs on the T2 image were defined as edema area according to previous literature [24, 25]. A third senior neuroradiologist with 10 years work experience then reevaluated the ROIs and made final decisions in cases where there was a lack of consensus.

Table 1 Patient characteristics

\begin{tabular}{llll}
\hline & $\begin{array}{l}\text { Training } \\
(n=160)\end{array}$ & $\begin{array}{l}\text { Validation } \\
(n=79)\end{array}$ & $p$ value \\
\hline Age (years; mean) & 43.0 & 42.5 & $0.736^{\mathrm{a}}$ \\
Sex (male/female) & $104 / 56$ & $53 / 26$ & $0.749^{\mathrm{b}}$ \\
Grade II/Grade III/Grade IV & $75 / 43 / 42$ & $39 / 23 / 17$ & $0.725^{\mathrm{b}}$ \\
Low VEGF/High VEGF & $63 / 97$ & $27 / 52$ & $0.435^{\mathrm{b}}$ \\
Tumor location left/right & $87 / 73$ & $42 / 37$ & $0.860^{\mathrm{b}}$ \\
\hline
\end{tabular}

${ }^{\mathrm{a}}$ Student's $\mathrm{t}$-test, ${ }^{\mathrm{b}} \mathrm{Chi}$-square test 


\section{Feature extraction}

To reduce bias due to data heterogeneity, the intensities of the voxels in each image were normalized to the $\mathrm{z}$ distribution. The slice thickness of MRI was resampled to $1 \mathrm{~mm}$ before feature extraction. Extraction of quantitative radiomic features was conducted as previously described [26], and the detailed equation pertaining to each feature is presented as Additional file 3 in that previous report. The feature extraction happened in 3D, and as a forward pass. We only extract radiomic features from preoperative T2 sequences, and a total of 431 radiomic features were obtained. The feature set included 14 first order statistics (pertaining to the distribution of signal intensity of images), 8 shape and sizebased features (quantifying the shape and size of tumors), 33 textural features (pertaining to intratumoral heterogeneity), and 376 wavelet features that were derived from group 1 and group 3 features via wavelet decomposition (using directional low-pass and high-pass filtering, the original feature was decomposed into 8 decompositions). All feature extraction processes were conducted using software developed in-house and implemented in MATLAB (2014a). The detailed function of the radiomic features are listed in the Additional file 3, and the extracted features are in compliance with the Image Biomarker Standardization Initiative [27, 28].

\section{Immunohistochemistry}

VEGF expression levels were evaluated by an eight-year work experience pathologist using typical tumor samples collected from the patients. Immunostaining was performed using an anti-VEGF antibody (Santa Cruz Biotechnology, Santa Cruz, CA) at a dilution of 1:100 in accordance with the manufacturer's instructions. Briefly, formalin-fixed paraffin-embedded tissue sections were cut into $5-\mu \mathrm{m}$ sections, which were then dried, dewaxed in xylene, rinsed in graded ethanol, and rehydrated in double-distilled water. Two pathologists who were blind to the clinical data scored the degree of staining. VEGF expression level was scored according to clinical practice: $(-)$ represented no or rare expression ( $<5 \%$ positive cells); $(+)$ represented mild expression (6-25\% positive cells); $(++)$ represented moderate expression (26-50\% positive cells); and $(+++)$ represented strong expression ( $>50 \%$ positive cells). Low VEGF expression was defined as VEGF ( - and + ), and high VEGF expression was defined as VEGF $(++$ and +++$)$ [29].

\section{Feature selection and classification}

To establish the radiomics model, the minimum redundancy maximum relevance (mRMR) algorithm was applied to select a subset of features from the 431 extracted radiomic features. The mRMR algorithm is an efficient data screening tool that has been widely used in many previous studies [30, 31]. In addition, we utilized a support vector machine (SVM) classifier to establish a machine-learning model for VEGF prediction. The SVM classifier is a widely used pattern recognition tool [32, 33]. Based on 10-fold cross validation, the parameters of SVM classifier were determined with the grid-search: kernel $=$ radial, gamma $=\left\{10^{-4}, 10^{-3}, 10^{-2}, 10^{-1}, 1,10,10^{2}, 10^{3}\right.$, $\left.10^{4}\right\}$, cost $=\left\{10^{-4}, 10^{-3}, 10^{-2}, 10^{-1}, 1,10,10^{2}, 10^{3}, 10^{4}\right\}$. Via these methods, we established a radiomics-based signature prediction model using data from the training group, and applied the same model in the validation group. A radiomics analysis protocol is shown in Fig. 1.

\section{Statistical analysis}

Statistical analyses and figure generation were mainly performed using R software (version 3.3.2; https://www.r-project.org/). The mRMR and SVM algorithms were conducted using "mRMRe" and "e1071" packages, respectively. Receiver operating characteristic (ROC) curves, and correlation heatmaps were depicted using "pROC" and "corrplot" packages, respectively. Differences between clinical characteristics were evaluated using the Chisquare test or Student's $t$-test.

\section{Results}

\section{Feature selection and classification}

In the current study, an efficient feature selection tool known as the mRMR algorithm was used, and a subset of 9 features were screened from a total of 431 radiomic features. The names and descriptions of these 9 selected features are shown in Table 2.

According to the cross-validation process, the SVM classifier performed best when the parameter gamma $=10^{-3}$ and cost $=10^{-3}$. Based on the selected radiomic features and the SVM classifier, a VEGF predictive machinelearning model was built using data derived from the training group. The areas under curve (AUC) were 0.741 in the training group (Fig. 2a) and 0.702 in the validation group (Fig. 2b). In ROC curve analysis, in the training group the optimal cutoff point of -0.356 exhibited respective sensitivity, specificity, and accuracy values of $83.5,58.7$, and $71.3 \%$, and in validation group the optimal cutoff point of -0.570 yielded corresponding values of $67.9,70.6$, and $72.3 \%$. Hence, the 9 selected radiomics features could be regarded as a VEGF-related signature, and the model established via mRMR and SVM algorithms in the training group exhibited effective performance in the validation group.

\section{Correlations between selected features}

Correlations between the VEGF-related features in the training group and the validation group are shown in Fig. 3. Although some of the 9 features exhibited high positive and negative correlations, the overall correlations between the features were low (mean \pm standard deviation $=0.112 \pm$ 0.029 in the training group and $0.174 \pm 0.052$ in the 


\section{Tumor segmentation \\ Radiomic feature extraction \\ Analysis}

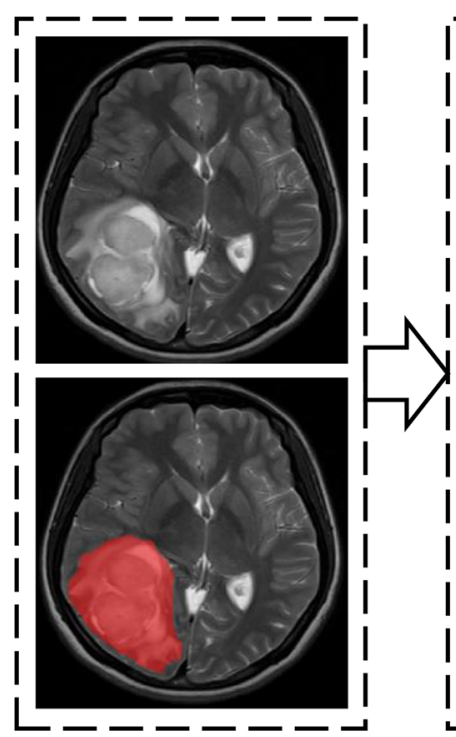

First order statistics

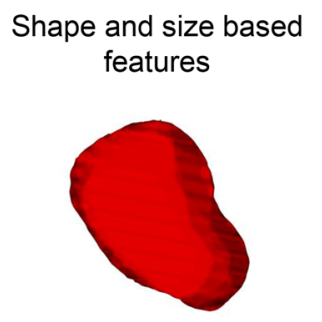

Textural features
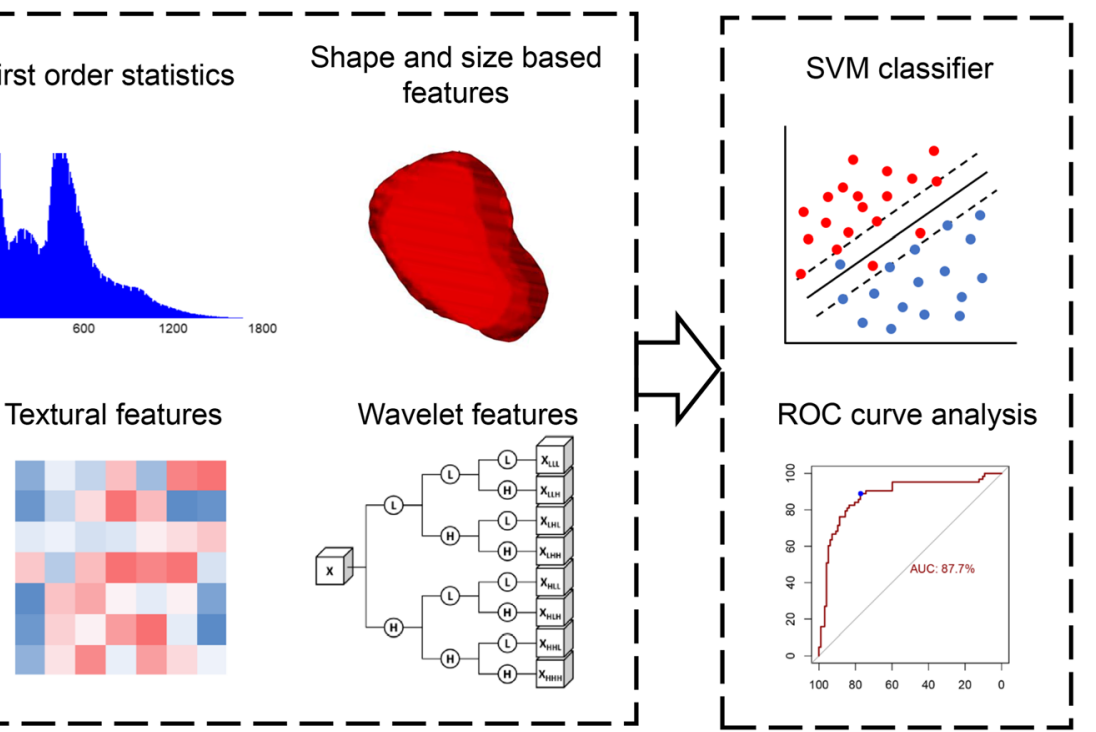

Fig. 1 The radiomics protocol. After the acquisition of magnetic resonance images, tumor segmentation was conducted using the image data. High-throughput radiomic features were then extracted from the segmented tumors, and further radiomic analysis was performed using these extracted features. SVM, support vector machine; ROC, receiver operating characteristic

validation group), suggesting that the features were independent of each other.

\section{Discussion}

In the current study, VEGF-related radiological signatures were analyzed via feature data extracted from T2weighted images of patients with diagnosed gliomas. Image features were extracted from each sequence and used to establish a radiomics-based model to predict VEGF expression levels via an mRMR algorithm and SVM classifier. The model achieved AUCs of $74.1 \%$ in the training group and $70.2 \%$ in the validation group. Overall, the results suggest that VEGF expression can be predicted using non-invasive radiological data, and that a machinelearning approach that integrates multivariate features is more effective than using individual features.

It has been reported that VEGF status was associated with some imaging features [29, 34-38]. A previous study reported that gliomas of different VEGF status tended to be situated at different locations [39]. They revealed that

Table 2 Nine radiomic features selected by the minimum redundancy maximum relevance algorithm

\begin{tabular}{|c|c|c|}
\hline Number & Features & Description \\
\hline 1 & Cluster Tendency_HLL & $\begin{array}{l}\text { One of the wavelet features derived from Cluster Tendency. Cluster Tendency is a measure of } \\
\text { groupings of voxels with similar gray-level values. }\end{array}$ \\
\hline 2 & Entropy_LLL (group 1 derived) & $\begin{array}{l}\text { One of the wavelet features derived from Entropy. Entropy specifies the uncertainty/randomness } \\
\text { in the image values. }\end{array}$ \\
\hline 3 & Long Run Low Gray Level Emphasis_LHL & $\begin{array}{l}\text { One of the wavelet features derived from Long Run Low Gray Level Emphasis. Long Run Low } \\
\text { Gray Level Emphasis measures the joint distribution of long runs and low gray level values. }\end{array}$ \\
\hline 4 & Minimum & Minimum describes the minimum signal intensity. \\
\hline 5 & Short Run High Gray Level Emphasis_LLH & $\begin{array}{l}\text { One of the wavelet features derived from Short Run High Gray Level Emphasis. Short Run High } \\
\text { Gray Level Emphasis measures the joint distribution of short runs and high gray level values. }\end{array}$ \\
\hline 6 & Short Run Low Gray Level Emphasis_LLL & $\begin{array}{l}\text { One of the wavelet features derived from Short Run Low Gray Level Emphasis. Short Run Low } \\
\text { Gray Level Emphasis measures the joint distribution of short runs and low gray level values. }\end{array}$ \\
\hline 7 & Short Run Low Gray Level Emphasis_LHH & $\begin{array}{l}\text { One of the wavelet features derived from Short Run Low Gray Level Emphasis. Short Run Low } \\
\text { Gray Level Emphasis measures the joint distribution of short runs and low gray level values. }\end{array}$ \\
\hline 8 & Short Run Low Gray Level Emphasis_HLL & $\begin{array}{l}\text { One of the wavelet features derived from Short Run Low Gray Level Emphasis. Short Run Low } \\
\text { Gray Level Emphasis measures the joint distribution of short runs and low gray level values. }\end{array}$ \\
\hline 9 & Short Run Low Gray Level Emphasis_HLH & $\begin{array}{l}\text { One of the wavelet features derived from Short Run Low Gray Level Emphasis. Short Run Low } \\
\text { Gray Level Emphasis measures the joint distribution of short runs and low gray level values. }\end{array}$ \\
\hline
\end{tabular}


a

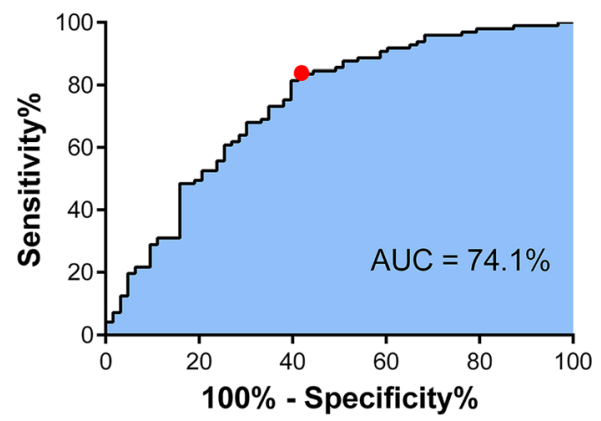

b

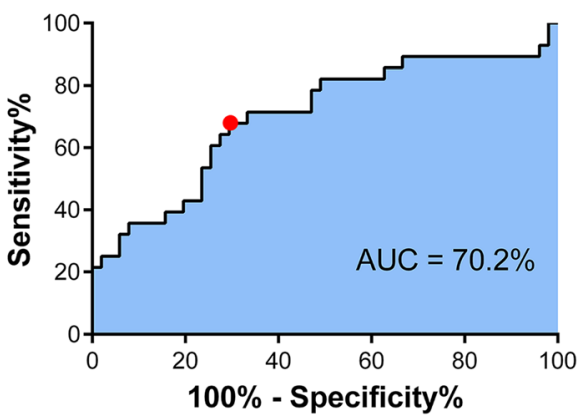

Fig. 2 Receiver operating characteristic curves for vascular endothelial growth factor status prediction in the training group and the validation group. a In the training group, the area under the curve was $74.1 \%$. At the optimal cutoff value (-0.356), the respective sensitivity, specificity, and accuracy values were $83.5,58.7$, and $71.3 \%$ (red dot). $\mathbf{b}$ In the validation set, the area under the curve was $70.2 \%$ and the optimal cutoff value (-0.570) exhibited respective sensitivity, specificity, and accuracy values of 67.9, 70.6, and 72.3\% (red dot). AUC, area under the curve

glioblastomas with high VEGF expression were more likely located in the left frontal lobe and the right caudate and these with low VEGF expression were more frequently located in the posterior region of the right lateral ventricle. We validated their findings on 59 patients in our study cohort, since only the 59 patients were diagnosed as glioblastoma. As shown in Additional file 2: Figure S2, the overall predictive accuracy was $60 \%$. Notably, there were 11 patients with tumors that did not involve left frontal lobe or the right caudate or the posterior region of the right lateral ventricle, which could not be classified. This kind of condition would limit the application of this method.
Moreover, Awasthi $\mathrm{R}$ et al. found that relative cerebral blood volume and relative cerebral blood flow were significantly correlated with VEGF expression level [38]; Li $\mathrm{K}$ et al. reported that combining the radiomic features and VEGF expression level could predict pelvic lymphatic metastasis [34]; Yin Q et al. revealed the associations between tumor angiogenesis and radiomic imaging features from PET/MRI [35], and Beig $\mathrm{N}$ et al. analyzed the hypoxia pathway radiomic features and predicted the overall survival in GBM [36]. These studies show that there were some radiological features that were significantly associated with VEGF expression status, but they

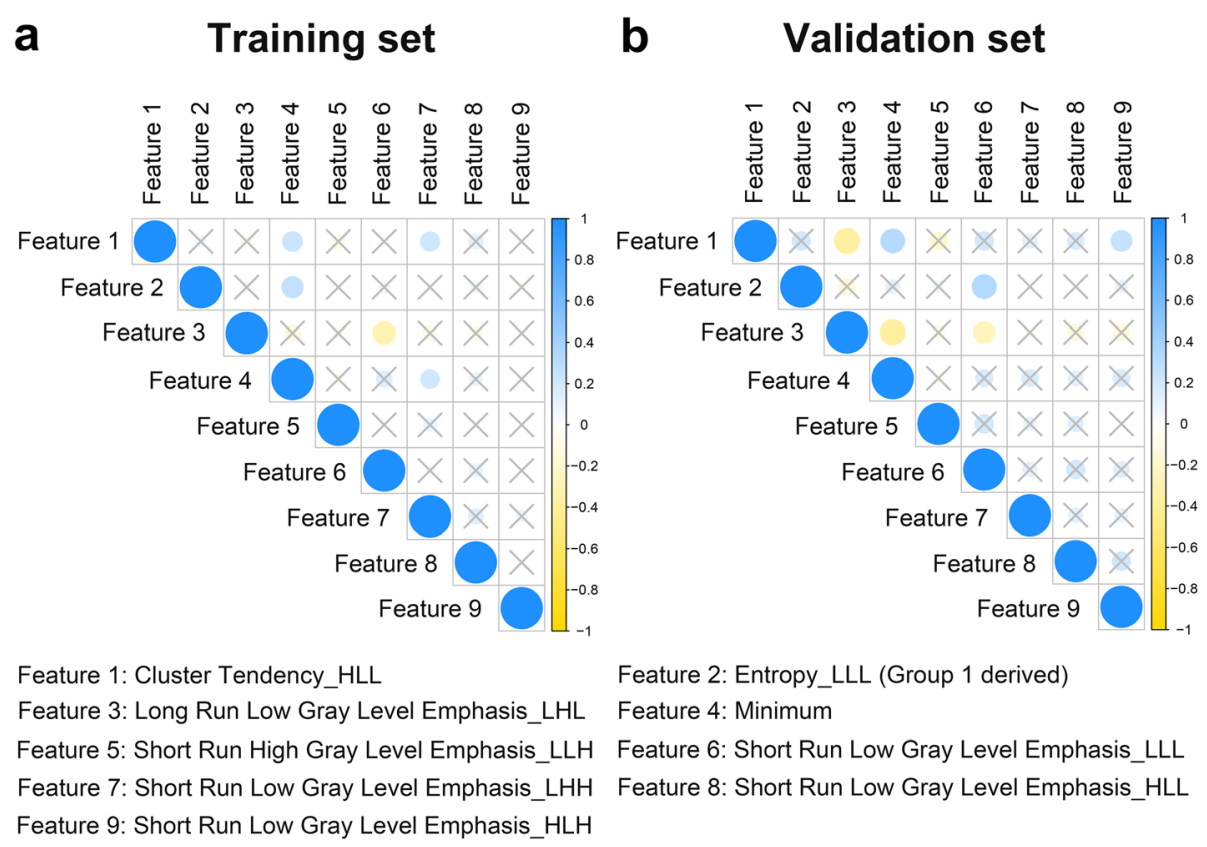

Fig. 3 Correlations between the 9 radiomic features that constituted the vascular endothelial growth factor-associated radiomic signature in the training and validation groups. Yellow dots indicate positive correlations and blue dots indicate negative correlations. Different sizes and color depths of dots indicate different correlation coefficients. Dots covered with crosses indicate non-significant correlations $(p>0.05)$ 
did not use independent validation data-sets to evaluate their findings, and their predictive power is limited. In recent years, radiomic analysis combined with algorithms has been widely used in radiology studies, and constitutes an efficient tool for studying relationships between images and tumors [11]. Many researchers have proved that radiomics models can predict molecule expression status efficiently [40-42]. Radiomics was also used to predict the anti-angiogenic treatment response [37]. We believe that there are clinically useful relationships between radiomic features and VEGF expression status.

The model established in the present study performed effectively in both the training group and the validation group, affirming the hypothesis that radiomics can predict VEGF expression level in tumor tissues. A previous study indicated that entropy could serve as an indicator for intra-tumoral heterogeneity and the degree of tumor malignancy [43], which could be used as an example to explain the association between radiomic features and VEGF expression. In addition, previous studies have shown that glioma patients with high expression of VEGF are more likely to have tissue edema [44-47]. T2 sequences can reflect tumors and tissue edema more accurately $[48,49]$. This may be the reason why the features selected based on T2 sequences could effectively establish a VEGF prediction model.

To establish the radiomics model, the mRMR algorithm was applied to select a subset of 9 features from the 431 extracted radiomic features. The mRMR algorithm is an efficient data dimensionality reduction algorithm for finding a set of both relevant and independent features that is widely used in bioinformation analysis [30, 31, 50]. The curse of dimensionality could be solved using the mRMR algorithm. The SVM classifier is an effective tool that exhibits better performance than other algorithms with regard to pattern recognition [32, 51, 52]. In the present study, the mRMR algorithm was used in combination with the SVM classifier to develop a method capable of effectively predicting VEGF expression status in glioblastoma patients.

The current study had some limitations. All the images were generated at a single center, so a multi-center study needs to be conducted in the future to investigate the universality of the model. Additionally, the correlative nature of the reported radiomic signature to VGEF expression needs to be further investigated by comparing to tissue biopsy results. It was also a retrospective study, so a prospective study is needed to verify the accuracy of the prediction model. Cases are manually annotated. In our current work we are trying to use an automated segmented approach that can level up the speed of radiomic pipeline. Next, we would collect more MRI protocols such as T1W and FLAIR (additionally also MRS, DTI) in our further work to build an advanced model. Finally, MRI scans have not been post-processed to a standard atlas, which might make the reproducibility of results difficult.

\section{Conclusion}

In conclusion, in the present study there were significant correlations between VEGF expression level and radiomic features in gliomas. Using the mRMR algorithm and SVM classifier, a VEGF expression level radiomic signature was developed, and VEGF expression level was effectively predicted in both a training group and a validation group. Radiogenomic analysis showed that VEGF expression level could also be reflected by the radiomic signature extracted from radiological images, indicating that the radiomic approach could potentially be a noninvasive surrogate indicator of gene expression level, and further assist patient-tailored treatment.

\section{Supplementary information}

Supplementary information accompanies this paper at https://doi.org/10. 1186/s40644-019-0256-y.

Additional file 1: Figure S1. Flow diagram of patients included and excluded in the final analysis.

Additional file 2: Figure S2. The prediction results of VEGF expression status in our cohort. The blue bars indicate that the predicted VEGF expression status was in accordance with the true VEGF expression status. The yellow bars indicate that the predicted VEGF expression status is not consistent with the true VEGF expression status.

Additional file 3. The feature extraction method used in the current study.

\section{Abbreviations}

AUC: Areas under curve; MRI: Magnetic resonance imaging; mRMR: the minimum redundancy maximum relevance algorithm; ROC: Receiver operating characteristic curves; ROI: Regions of interest; SVM: Support vector machine; T2: T2-weighted; VEGF: Vascular endothelial growth factor

\section{Acknowledgements}

Not applicable.

\section{Ethical approval}

This study has been approved by the local ethics committee and has been performed in accordance with the ethical standards laid down in the 1964 Declaration of Helsinki and its later amendments.

Conflict of interest

The authors declare that they have no conflict of interest.

\section{Authors' contributions}

Study concept and design: XL and TJ; All the authors contributed to the data analysis and interpretation. ZYS and YML performed the statistical analysis; ZYS, YYW and XF were major contributors and contributed equally to writing the manuscript. KX, KW extracted the texture features. SWL, ZZ and TJ contributed to the MRI data acquisition. All authors have read and approved the final manuscript.

\section{Funding}

This study was funded by the National Natural Science Foundation of China (No. 81601452), the Beijing Natural Science Foundation (No. 7174295), the National Key Research and Development Plan (No. 2016YFC0902500).

\section{Availability of data and materials}

The datasets supporting the conclusion of this article available from the corresponding author on reasonable request. 


\section{Consent for publication}

Written informed consent was obtained from each patient.

\begin{abstract}
Author details
'Beijing Neurosurgical Institute, Capital Medical University, 6 Tiantanxili, Beijing 100050, China. ${ }^{2}$ Department of Neurosurgery, Beijing Tiantan Hospital, Capital Medical University, Beijing, China. ${ }^{3}$ Chinese Academy of Sciences, Institute of Automation, Beijing, China. ${ }^{4}$ Department of Nuclear Medicine, Beijing Tiantan Hospital, Capital Medical University, Beijing, China. ${ }^{5}$ Center of Brain Tumor, Beijing Institute for Brain Disorders, Beijing, China. ${ }^{6} \mathrm{C}$ China National Clinical Research Center for Neurological Diseases, Beijing, China. ${ }^{7}$ Chinese Glioma Genome Atlas Network (CGGA) and Asian Glioma Genome Atlas Network (AGGA), Beijing, China.
\end{abstract}

Received: 22 March 2019 Accepted: 25 September 2019 Published online: 21 October 2019

\section{References}

1. Ostrom QT, Gittleman H, Liao P, Vecchione-Koval T, Wolinsky Y, Kruchko C, Barnholtz-Sloan JS. CBTRUS Statistical Report: Primary brain and other central nervous system tumors diagnosed in the United States in 20102014. Neuro-oncology. 2017;19(suppl_5):v1-v88.

2. Louis DN, Perry A, Reifenberger G, von Deimling A, Figarella-Branger D, Cavenee WK, Ohgaki H, Wiestler OD, Kleihues P, Ellison DW. The 2016 World Health Organization classification of tumors of the central nervous system: a summary. Acta Neuropathol. 2016;131(6):803-20.

3. Nabors LB, Portnow J, Ammirati M, Baehring J, Brem H, Butowski $N$, Fenstermaker RA, Forsyth P, Hattangadi-Gluth J, Holdhoff M, et al. NCCN guidelines insights: central nervous system cancers, version 1.2017. J Natl Compr Cancer Netw. 2017:15(11):1331-45.

4. Turkowski K, Brandenburg S, Mueller A, Kremenetskaia I, Bungert AD, Blank $A$, Felsenstein $M$, Vajkoczy P. VEGF as a modulator of the innate immune response in glioblastoma. Glia. 2018;66(1):161-74.

5. Chen W, He D, Li Z, Zhang X, Pan D, Chen G. Overexpression of vascular endothelial growth factor indicates poor outcomes of glioma: a systematic review and meta-analysis. Int J Clin Exp Med. 2015;8(6):8709-19.

6. Chaudhry $\mathrm{H}$, O'Donovan DG, Brenchley PE, Reid H, Roberts IS. Vascular endothelial growth factor expression correlates with tumour grade and vascularity in gliomas. Histopathology. 2001;39(4):409-15.

7. Lu-Emerson C, Duda DG, Emblem KE, Taylor JW, Gerstner ER, Loeffler JS, Batchelor TT, Jain RK. Lessons from anti-vascular endothelial growth factor and anti-vascular endothelial growth factor receptor trials in patients with glioblastoma. J Clin Oncol. 2015;33(10):1197-213.

8. Wirsching HG, Weiss T, Roth P. Weller M: [basic principles of diagnosis and treatment of gliomas]. Nervenarzt. 2018.

9. Pope WB, Chen JH, Dong J, Carlson MR, Perlina A, Cloughesy TF, Liau LM, Mischel PS, Nghiemphu P, Lai A, et al. Relationship between gene expression and enhancement in glioblastoma multiforme: exploratory DNA microarray analysis. Radiology. 2008;249(1):268-77.

10. Gillies RJ, Kinahan PE, Hricak H. Radiomics: images are more than pictures They Are Data. Radiology. 2016;278(2):563-77.

11. Lambin P, Rios-Velazquez E, Leijenaar R, Carvalho $S$, van Stiphout RG, Granton P, Zegers CM, Gillies R, Boellard R, Dekker A, et al. Radiomics: extracting more information from medical images using advanced feature analysis. Eur J Cancer. 2012;48(4):441-6.

12. Herold CJ, Lewin JS, Wibmer AG, Thrall JH, Krestin GP, Dixon AK, Schoenberg SO, Geckle RJ, Muellner A, Hricak H. Imaging in the age of precision medicine: summary of the proceedings of the 10th biannual symposium of the International Society for Strategic Studies in radiology. Radiology. 2016;279(1):226-38.

13. Parliament MB. Radiogenomics: associations in all the wrong places? Lancet Oncol. 2012;13(1):7-8.

14. Cha KH, Hadjiiski L, Chan HP, Weizer AZ, Alva A, Cohan RH, Caoili EM, Paramagul C, Samala RK. Bladder Cancer treatment response assessment in CT using Radiomics with deep-learning. Sci Rep. 2017;7(1):8738.

15. Ferreira Junior JR, Koenigkam-Santos M, Cipriano FEG, Fabro AT, AzevedoMarques PM. Radiomics-based features for pattern recognition of lung cancer histopathology and metastases. Comput Methods Prog Biomed. 2018;159:23-30.

16. Bak SH, Park H, Lee HY, Kim Y, Kim HL, Jung SH, Kim H, Kim J, Park K. Imaging genotyping of functional signaling pathways in lung squamous cell carcinoma using a radiomics approach. Sci Rep. 2018;8(1):3284.
17. Chang K, Bai HX, Zhou H, Su C, Bi WL, Agbodza E, Kavouridis VK, Senders JT, Boaro A, Beers A, et al. Residual convolutional neural network for the determination of IDH status in low- and high-grade gliomas from MR imaging. Clin Cancer Res. 2018;24(5):1073-81.

18. Chang P, Grinband J, Weinberg BD, Bardis M, Khy M, Cadena G, Su MY, Cha S, Filippi CG, Bota D, et al. Deep-learning convolutional neural networks accurately classify genetic mutations in gliomas. AJNR Am J Neuroradiol. 2018;39(7):1201-7.

19. Binder ZA, Thorne AH, Bakas S, Wileyto EP, Bilello M, Akbari H, Rathore S, Ha SM, Zhang L, Ferguson CJ, et al. Epidermal growth factor receptor extracellular domain mutations in glioblastoma present opportunities for clinical imaging and therapeutic development. Cancer Cell. 2018;34(1):163-77 e167.

20. Akbari H, Bakas S, Pisapia JM, Nasrallah MP, Rozycki M, Martinez-Lage M, Morrissette JJD, Dahmane N, O'Rourke DM, Davatzikos C. In vivo evaluation of EGFRvIII mutation in primary glioblastoma patients via complex multiparametric MRI signature. Neuro-Oncology. 2018;20(8):1068-79.

21. Li Y, Liu X, Xu K, Qian Z, Wang K, Fan X, Li S, Wang Y, Jiang T. MRI features can predict EGFR expression in lower grade gliomas: a voxel-based radiomic analysis. Eur Radiol. 2018;28(1):356-62.

22. Li Y, Qian Z, Xu K, Wang K, Fan X, Li S, Liu X, Wang Y, Jiang T. Radiomic features predict Ki-67 expression level and survival in lower grade gliomas. J Neuro-Oncol. 2017;135(2):317-24

23. Wu S, Meng J, Yu Q, Li P, Fu S. Radiomics-based machine learning methods for isocitrate dehydrogenase genotype prediction of diffuse gliomas. J Cancer Res Clin Oncol. 2019;145(3):543-50.

24. Menze BH, Jakab A, Bauer S, Kalpathy-Cramer J, Farahani K, Kirby J, Burren Y Porz N, Slotboom J, Wiest R, et al. The multimodal brain tumor image segmentation benchmark (BRATS). IEEE Trans Med Imaging. 2015;34(10): 1993-2024.

25. Bakas S, Akbari H, Sotiras A, Bilello M, Rozycki M, Kirby JS, Freymann JB, Farahani K, Davatzikos C. Advancing the Cancer genome atlas glioma MRI collections with expert segmentation labels and radiomic features. Sci Data. 2017:4:170117.

26. Aerts HJ, Velazquez ER, Leijenaar RT, Parmar C, Grossmann P, Carvalho S, Bussink J, Monshouwer R, Haibe-Kains B, Rietveld D, et al. Decoding tumour phenotype by noninvasive imaging using a quantitative radiomics approach. Nat Commun. 2014;5:4006.

27. Lambin $\mathrm{P}$, Leijenaar RTH, Deist TM, Peerlings J, de Jong EEC, van Timmeren J, Sanduleanu S, Larue R, Even AJG, Jochems A, et al. Radiomics: the bridge between medical imaging and personalized medicine. Nat Rev Clin Oncol. 2017;14(12):749-62

28. Lambin P. Radiomics digital phantom. CancerData. 2016

29. Wang Y, Wang K, Li H, Wang J, Wang L, Dai J, Jiang T, Ma J. Identifying the association of contrast enhancement with vascular endothelia growth factor expression in anaplastic gliomas: a volumetric magnetic resonance imaging analysis. PLoS One. 2015;10(3):e0121380.

30. Cai $Y$, Huang $T$, Hu L, Shi $X$, Xie L, Li Y. Prediction of lysine ubiquitination with mRMR feature selection and analysis. Amino Acids. 2012;42(4):1387-95.

31. Alshamlan H, Badr G, Alohali Y. mRMR-ABC: a hybrid gene selection algorithm for Cancer classification using microarray gene expression profiling. Biomed Res Int. 2015;2015:604910.

32. Fu MR, Wang Y, Li C, Qiu Z, Axelrod D, Guth AA, Scagliola J, Conley Y, Aouizerat BE, Qiu JM, et al. Machine learning for detection of lymphedema among breast cancer survivors. Mhealth. 2018;4:17.

33. Korfiatis $P$, Kline TL, Coufalova L, Lachance DH, Parney IF, Carter RE, Buckner JC, Erickson BJ. MRI texture features as biomarkers to predict MGMT methylation status in glioblastomas. Med Phys. 2016:43(6):2835.

34. Li K, Sun H, Lu Z, Xin J, Zhang L, Guo Y, Guo Q. Value of [(18) F] FDG PET radiomic features and VEGF expression in predicting pelvic lymphatic metastasis and their potential relationship in early-stage cervical squamous cell carcinoma. Eur J Radiol. 2018;106:160-6.

35. Yin Q, Hung SC, Wang L, Lin W, Fielding JR, Rathmell WK, Khandani AH, Woods ME, Milowsky MI, Brooks SA, et al. Associations between tumor vascularity Vascular Endothelial Growth Factor Expression and PET/MRI Radiomic Signatures in Primary Clear-Cell-Renal-Cell-Carcinoma: Proof-ofConcept Study. Scientific Reports. 2017;7:43356.

36. Beig N, Patel J, Prasanna P, Hill V, Gupta A, Correa R, Bera K, Singh S, Partovi $\mathrm{S}$, Varadan $\mathrm{V}$, et al. Radiogenomic analysis of hypoxia pathway is predictive of overall survival in glioblastoma. Sci Rep. 2018;8(1):7.

37. Kickingereder P, Gotz M, Muschelli J, Wick A, Neuberger U, Shinohara RT, Sill M, Nowosielski M, Schlemmer HP, Radbruch A, et al. Large-scale Radiomic 
profiling of recurrent glioblastoma identifies an imaging predictor for stratifying anti-Angiogenic treatment response. Clin Cancer Res. 2016;22(23): 5765-71.

38. Awasthi R, Rathore RK, Soni P, Sahoo P, Awasthi A, Husain N, Behari S, Singh RK, Pandey CM, Gupta RK. Discriminant analysis to classify glioma grading using dynamic contrast-enhanced MRI and immunohistochemical markers. Neuroradiol. 2012;54(3):205-13

39. Fan X, Wang Y, Wang K, Liu S, Liu Y, Ma J, Li S, Jiang T. Anatomical specificity of vascular endothelial growth factor expression in glioblastomas: a voxel-based mapping analysis. Neuroradiol. 2016;58(1):69-75.

40. Kotrotsou A, Zinn PO, Colen RR. Radiomics in brain tumors: an emerging technique for characterization of tumor environment. Magn Reson Imaging Clin N Am. 2016;24(4):719-29.

41. Yu J, Shi Z, Lian Y, Li Z, Liu T, Gao Y, Wang Y, Chen L, Mao Y. Noninvasive IDH1 mutation estimation based on a quantitative radiomics approach for grade II glioma. Eur Radiol. 2016.

42. Wang JW, Li JP, Wang YP, Zhang XH, Zhang YQ. Deep brain stimulation for myoclonus-dystonia syndrome with double mutations in DYT1 and DYT11. Sci Rep. 2017;7:41042.

43. Ganeshan B, Miles KA, Young RC, Chatwin CR. Texture analysis in noncontrast enhanced $C T$ : impact of malignancy on texture in apparently disease-free areas of the liver. Eur J Radiol. 2009:70(1):101-10.

44. Jain RK, di Tomaso E, Duda DG, Loeffler JS, Sorensen AG, Batchelor TT. Angiogenesis in brain tumours. Nat Rev Neurosci. 2007;8(8):610-22.

45. Norden AD, Drappatz J, Wen PY. Antiangiogenic therapies for high-grade glioma. Nat Rev Neurol. 2009;5(11):610-20

46. Carlson MR, Pope WB, Horvath S, Braunstein JG, Nghiemphu P, Tso CL, Mellinghoff I, Lai A, Liau LM, Mischel PS, et al. Relationship between survival and edema in malignant gliomas: role of vascular endothelial growth factor and neuronal pentraxin 2. Clin Cancer Res. 2007;13(9):2592-8.

47. Li X, Padhan N, Sjostrom EO, Roche FP, Testini C, Honkura N, Sainz-Jaspeado M, Gordon E, Bentley K, Philippides A, et al. VEGFR2 pY949 signalling regulates adherens junction integrity and metastatic spread. Nat Commun. 2016;7:11017.

48. Jensen RL, Mumert ML, Gillespie DL, Kinney AY, Schabel MC, Salzman KL. Preoperative dynamic contrast-enhanced MRI correlates with molecular markers of hypoxia and vascularity in specific areas of intratumoral microenvironment and is predictive of patient outcome. Neuro-oncology. 2014;16(2):280-91.

49. Pope WB, Young JR, Ellingson BM. Advances in MRl assessment of gliomas and response to anti-VEGF therapy. Curr Neurol Neurosci Rep. 2011;11(3):336-44.

50. Li BQ, Hu LL, Chen L, Feng KY, Cai YD, Chou KC. Prediction of protein domain with mRMR feature selection and analysis. PLoS One. 2012;7(6):e39308.

51. Zhang G, Piccardi M, Zare Borzeshi E. Sequential labeling with structural SVM under nondecomposable losses. IEEE Trans Neural Netw Learn Syst. 2017.

52. Hotzy F, Theodoridou A, Hoff P, Schneeberger AR, Seifritz E, Olbrich S, Jager M. Machine learning: an approach in identifying risk factors for coercion compared to binary logistic regression. Front Psychiatry. 2018;9:258.

\section{Publisher's Note}

Springer Nature remains neutral with regard to jurisdictional claims in published maps and institutional affiliations.

Ready to submit your research? Choose BMC and benefit from:

- fast, convenient online submission

- thorough peer review by experienced researchers in your field

- rapid publication on acceptance

- support for research data, including large and complex data types

- gold Open Access which fosters wider collaboration and increased citations

- maximum visibility for your research: over $100 \mathrm{M}$ website views per year

At BMC, research is always in progress.

Learn more biomedcentral.com/submissions 